\title{
HRRP target recognition based on kernel joint discriminant analysis
}

\author{
LIU Wenbo ${ }^{1, *}$, YUAN Jiawen ${ }^{1}$, ZHANG Gong ${ }^{2}$, and SHEN Qian ${ }^{1}$ \\ 1. College of Automation Engineering, Nanjing University of Aeronautics and Astronautics, Nanjing 211106, China; \\ 2. College of Electronic and Information Engineering, Nanjing University of Aeronautics and Astronautics, Nanjing 211106, China
}

\begin{abstract}
With the improvement of radar resolution, the dimension of the high resolution range profile (HRRP) has increased. In order to solve the small sample problem caused by the increase of HRRP dimension, an algorithm based on kernel joint discriminant analysis (KJDA) is proposed. Compared with the traditional feature extraction methods, KJDA possesses stronger discriminative ability in the kernel feature space. K-nearest neighbor (KNN) and kernel support vector machine (KSVM) are applied as feature classifiers to verify the classification effect. Experimental results on the measured aircraft datasets show that KJDA can reduce the dimensionality, and improve target recognition performance.
\end{abstract}

Keywords: high resolution range profile (HRRP), target recognition, small sample problem, feature extraction, dimension reduction.

DOI: $10.21629 / J S E E .2019 .04 .08$

\section{Introduction}

High resolution range profile (HRRP) reflects physical structure information through the distribution of target scattering centers along the radar line-of-sight. Therefore, HRRP plays an important role in radar automatic target recognition (RATR) [1 -4].

With the improvement of radar resolution, the target structure information contained in the HRRP becomes richer, which will burden the storage system and the classifier. In addition, when the number of samples is much smaller than its dimension, it will lead to the small sample problem [5]. Some linear feature extraction methods are adopted to resolve the above problems such as principal component analysis (PCA) [6,7], linear discriminant analysis (LDA) [8,9], and so on. They have strong advantages in dimension reduction. However, they are difficult to

Manuscript received April 17, 2018.

*Corresponding author.

This work was supported by the National Natural Science Foundation of China (61471191), the Aeronautical Science Foundation of China (20152052026), and the Foundation of Graduate Innovation Center in NUAA (kfjj20170313). extract nonlinear features from the original data. The nonlinear feature extraction methods can effectively reduce dimensionality and enhance separability between targets. Kernel LDA (KLDA) $[10,11]$ extracts the nonlinear discriminant features of the training samples and deal with the nonlinear classification availably. However, KLDA assumes that homogeneous samples strictly obey Gaussian distribution in kernel feature space. It is not suitable to assume that HRRP follows Gaussian distribution. Feng et al. [12] applied kernel tricks in local sampling mean discriminant analysis (KLSMDA) which achieved a high classification accuracy in HRRP target recognition. Nevertheless, KLSMDA is not applicable for solving the small sample problem.

In order to overcome the small sample problem and reduce dimensionality in HRRP target recognition, a new nonlinear feature extraction method named kernel joint discriminant analysis (KJDA) is proposed. Different from other feature extraction methods, it utilizes more potential information (information captured from global and local criterion of input data in kernel feature space), which may give extra support for strengthening recognition. In addition, it automatically seeks an appropriate compromise between global and local criterion based on the essential characteristics of the data, thereby weakening the interference caused by the small sample problem in HRRP target recognition.

This paper is organized as follows. In Section 2, we give a rough overview of KLSMDA. In Section 3, we demonstrate the principles of KJDA. The experimental results of HRRP target recognition are presented in Section 4. Finally, conclusions and future work are drawn in Section 5.

\section{Brief of KLSMDA}

KLSMDA is a feature extraction method optimized by local criterion where similarities are evaluated by distances between neighboring pairs. Assume $C$ is the number of the 
training data classes, each class is composed of $N_{i}(i=$ $1, \ldots, C)$ training samples, and $N=\sum_{i=1}^{C} N_{i}$ indicates the number of all training samples. Let $\boldsymbol{x}_{i}^{p} \in \mathbf{R}^{D}$ be a $D$ dimensional column vector of the $p$ th sample in the $i$ th class, then $\boldsymbol{x}_{i}^{p}$ can be projected to a higher dimensional feature space $\boldsymbol{H}$ by a mapping function $\phi: \boldsymbol{x}_{i}^{p} \in \mathbf{R}^{D} \rightarrow$ $\phi\left(\boldsymbol{x}_{i}^{p}\right) \in \boldsymbol{H}$.

Firstly, we introduce a definition of local sampling mean. Suppose that the affinity matrix $Q$ can be formed as

$$
\boldsymbol{Q}\left(\boldsymbol{x}_{i}^{p}, \boldsymbol{x}_{j}^{q}\right)=\exp \left(-\left\|\phi\left(\boldsymbol{x}_{i}^{p}\right)-\phi\left(\boldsymbol{x}_{j}^{q}\right)\right\|_{2}^{2} / \sigma_{1}\right)
$$

where $\sigma_{1}$ is the kernel parameter. Let $\boldsymbol{\mu}_{\left(\boldsymbol{x}_{i}^{p}, j\right)}^{\phi}=$ $\sum_{q=1}^{N_{j}} \boldsymbol{Q}\left(\boldsymbol{x}_{i}^{p}, \boldsymbol{x}_{j}^{q}\right) \phi\left(\boldsymbol{x}_{j}^{q}\right) / \sum_{q=1}^{N_{j}} \boldsymbol{Q}\left(\boldsymbol{x}_{i}^{p}, \boldsymbol{x}_{j}^{q}\right)$ be the local sampling mean of the sample $\boldsymbol{x}_{i}^{p}$ for the class $j$ in the kernel feature space $\boldsymbol{H}$. If $i=j, \boldsymbol{\mu}_{\left(\boldsymbol{x}_{i}^{p}, j\right)}^{\phi}$ represents $\boldsymbol{x}_{i}^{p}$ 's homogeneous local sampling mean in $\boldsymbol{H}$; otherwise it is a heterogeneous one.

Secondly, the within-class and between-class scattering matrices in the kernel space, $\boldsymbol{H}, \boldsymbol{S}_{B}^{\phi}$ and $\boldsymbol{S}_{W}^{\phi}$, can be defined as

$$
\begin{aligned}
\boldsymbol{S}_{B}^{\phi}= & \sum_{i=1}^{C} \sum_{p=1}^{N_{i}} \sum_{j=1, j \neq i}^{C} \gamma_{B\left(\boldsymbol{x}_{i}^{p}, j\right)}^{\phi}\left(\phi\left(\boldsymbol{x}_{i}^{p}\right)-\right. \\
& \left.\boldsymbol{\mu}_{\left(\boldsymbol{x}_{i}^{p}, j\right)}^{\phi}\right)\left(\phi\left(\boldsymbol{x}_{i}^{p}\right)-\boldsymbol{\mu}_{\left(\boldsymbol{x}_{i}^{p}, j\right)}^{\phi}\right)^{\mathrm{T}} \\
\boldsymbol{S}_{W}^{\phi}= & \sum_{i=1}^{C} \sum_{p=1}^{N_{i}} \gamma_{W\left(\boldsymbol{x}_{i}^{p}, i\right)}^{\phi}\left(\phi\left(\boldsymbol{x}_{i}^{p}\right)-\boldsymbol{\mu}_{\left(\boldsymbol{x}_{i}^{p}, i\right)}^{\phi}\right) .
\end{aligned}
$$

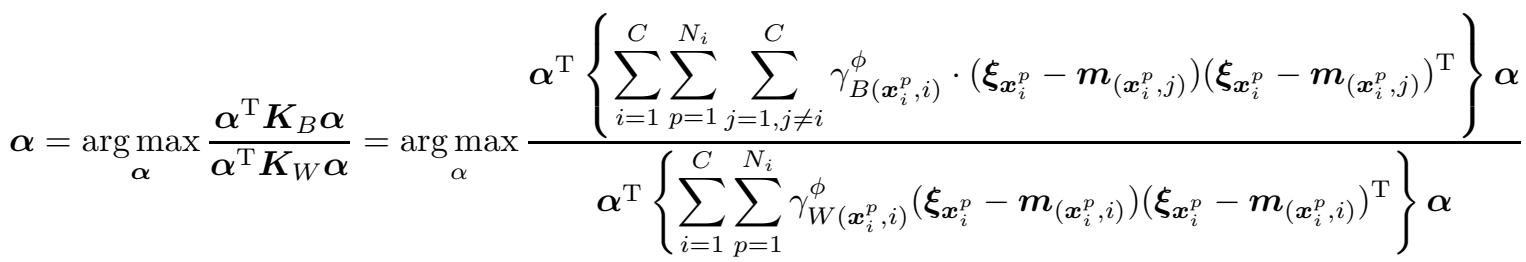

$$
\left(\phi\left(\boldsymbol{x}_{i}^{p}\right)-\boldsymbol{\mu}_{\left(x_{i}^{p}, i\right)}^{\phi}\right)^{\mathrm{T}}
$$

where $\gamma_{B\left(\boldsymbol{x}_{i}^{p}, j\right)}^{\phi}$ and $\gamma_{W\left(\boldsymbol{x}_{i}^{p}, i\right)}^{\phi}$ are, respectively, the weighting parameters to describe the separability of betweenclass and the similarity of within-class samples through discrepant vectors in the kernel feature space $\boldsymbol{H}$.

$$
\begin{gathered}
\gamma_{B\left(\boldsymbol{x}_{i}^{p}, j\right)}^{\phi}=\exp \left[\frac{1-\eta_{i j p}^{\phi}}{\sigma_{2}\left(1+\eta_{i j p}^{\phi}\right)}\right] \\
\gamma_{W\left(\boldsymbol{x}_{i}^{p}, i\right)}^{\phi}=\frac{1}{C-1} \sum_{j=1, j \neq i}^{C} \gamma_{B\left(\boldsymbol{x}_{i}^{p}, j\right)}^{\phi} \\
\eta_{i j p}^{\phi}=\left\|\phi\left(\boldsymbol{x}_{i}^{p}\right)-\boldsymbol{\mu}_{\left(\boldsymbol{x}_{i}^{p}, j\right)}^{\phi}\right\|_{2} /\left\|\phi\left(\boldsymbol{x}_{i}^{p}\right)-\boldsymbol{\mu}_{\left(\boldsymbol{x}_{i}^{p}, i\right)}^{\phi}\right\|_{2}
\end{gathered}
$$

where $\sigma_{2}$ is the parameter that controls the varying speed of $\gamma_{B\left(\boldsymbol{x}_{i}^{p}, j\right)}^{\phi}$. On the summary of the above formulas, KLSMDA can be defined as

$$
\boldsymbol{v}=\underset{\boldsymbol{v}}{\arg \max } \frac{\boldsymbol{v}^{\mathrm{T}} \boldsymbol{S}_{B}^{\phi} \boldsymbol{v}}{\boldsymbol{v}^{\mathrm{T}} \boldsymbol{S}_{W}^{\phi} \boldsymbol{v}}
$$

where $\boldsymbol{v}$ can nonlinearly project data $\boldsymbol{x}_{p}^{i}$ onto a lower $d$ dimensional column vector $\boldsymbol{y}_{p}^{i}=\boldsymbol{v}^{\mathrm{T}} \phi\left(\boldsymbol{x}_{p}^{i}\right) \in \mathbf{R}^{d}(d \ll$ $D)$. From the theory of reproducing kernels [13], we can find an expansion for $v$ of the form:

$$
\begin{gathered}
\boldsymbol{v}=\sum_{i=1}^{C} \sum_{p=1}^{N_{i}} \alpha_{p} \phi\left(\boldsymbol{x}_{p}^{i}\right)=\phi(\boldsymbol{X}) \boldsymbol{\alpha} \\
\text { s.t. } \boldsymbol{\alpha}=\left[\alpha_{1}^{1}, \ldots, \alpha_{C}^{N_{C}}\right]^{\mathrm{T}} .
\end{gathered}
$$

Using (7), (6) can be changed as where $\boldsymbol{\xi}_{\boldsymbol{x}_{i}^{p}}=\left[k\left(\boldsymbol{x}_{i}^{p}, \boldsymbol{x}_{1}^{1}\right), \ldots, k\left(\boldsymbol{x}_{i}^{p}, \boldsymbol{x}_{C}^{N_{C}}\right)\right]^{\mathrm{T}} \in \mathbf{R}^{N}$ with the introduced kernel function $k(\cdot, \cdot)[14] ; \boldsymbol{m}_{\left(\boldsymbol{x}_{i}^{p}, j\right)}=$ $\sum_{q=1}^{N_{j}} Q\left(\boldsymbol{x}_{i}^{p}, \boldsymbol{x}_{j}^{q}\right) \boldsymbol{\xi}_{\boldsymbol{x}_{i}^{p}} / \sum_{q=1}^{N_{j}} Q\left(\boldsymbol{x}_{i}^{p}, \boldsymbol{x}_{j}^{q}\right)$.

Finally, (8) can be resolved by utilizing generalized eigenvalue decomposition $\boldsymbol{K}_{B} \boldsymbol{\alpha}=\lambda \boldsymbol{K}_{W} \boldsymbol{\alpha}$. Then, a $d$ dimensional column vector $\boldsymbol{y}$ is expressed as

$$
\boldsymbol{y}=\boldsymbol{v}^{\mathrm{T}} \phi(\boldsymbol{x})=\boldsymbol{\alpha}^{\mathrm{T}}\left[k\left(\boldsymbol{x}, \boldsymbol{x}_{1}^{1}\right), \ldots, k\left(\boldsymbol{x}, \boldsymbol{x}_{C}^{N_{C}}\right)\right]^{\mathrm{T}} .
$$

\section{Principles of KJDA}

In this section, we introduce the KJDA method to make up for the drawbacks of the small sample problem and reduce dimensionality in HRRP target recognition.

Different from KLSMDA and KLDA, KJDA is a feature extraction method combing global criterion and local criterion. Firstly, we introduce definitions of global and local sampling mean. Let $\boldsymbol{\mu}_{i}^{\phi}=\frac{1}{N_{i}} \sum_{p=1}^{N_{i}} \phi\left(\boldsymbol{x}_{i}^{p}\right)$ and 
$\boldsymbol{\mu}^{\phi}=\frac{1}{N} \sum_{i=1}^{C} \sum_{p=1}^{N_{i}} \phi\left(\boldsymbol{x}_{i}^{p}\right)$ represent the global sampling mean of the $i$ th and all classes in the kernel feature space $\boldsymbol{H}$, respectively. The definition of the local sampling mean $\boldsymbol{\mu}_{\left(\boldsymbol{x}_{i}^{p}, j\right)}^{\phi}$ in KJDA is in common with the definition in KLSMDA.

Secondly, the global within-class scattering matrix $S_{G B}^{\phi}$, the global between-class one $\boldsymbol{S}_{G W}^{\phi}$, the local withinclass one $\boldsymbol{S}_{L B}^{\phi}$, and the local between-class one $\boldsymbol{S}_{L W}^{\phi}$ in the kernel feature space $\boldsymbol{H}$ are defined respectively as follows:

$$
\begin{gathered}
\boldsymbol{S}_{G B}^{\phi}=\sum_{i=1}^{C}\left(\boldsymbol{\mu}_{i}^{\phi}-\boldsymbol{\mu}^{\phi}\right)\left(\boldsymbol{\mu}_{i}^{\phi}-\boldsymbol{\mu}^{\phi}\right)^{\mathrm{T}} \\
\boldsymbol{S}_{G W}^{\phi}=\sum_{i=1}^{C} \sum_{p=1}^{N_{i}}\left(\phi\left(\boldsymbol{x}_{i}^{p}\right)-\boldsymbol{\mu}_{i}^{\phi}\right) \cdot\left(\phi\left(\boldsymbol{x}_{i}^{p}\right)-\boldsymbol{\mu}_{i}^{\phi}\right)^{\mathrm{T}} \\
\boldsymbol{S}_{L B}^{\phi}=\sum_{i=1}^{C} \sum_{p=1}^{N_{i}} \sum_{j=1, j \neq i}^{C} \gamma_{B\left(\boldsymbol{x}_{i}^{p}, j\right)}^{\phi} \\
\left(\phi\left(\boldsymbol{x}_{i}^{p}\right)-\boldsymbol{\mu}_{\left(\boldsymbol{x}_{i}^{p}, j\right)}^{\phi}\right)\left(\phi\left(\boldsymbol{x}_{i}^{p}\right)-\boldsymbol{\mu}_{\left(\boldsymbol{x}_{i}^{p}, j\right)}^{\phi}\right)^{\mathrm{T}} \\
\boldsymbol{S}_{L W}^{\phi}=\sum_{i=1}^{C} \sum_{p=1}^{N_{i}} \gamma_{W\left(\boldsymbol{x}_{i}^{p}, i\right)}^{\phi}\left(\phi\left(\boldsymbol{x}_{i}^{p}\right)-\right. \\
\left.\boldsymbol{\mu}_{\left(\boldsymbol{x}_{i}^{p}, i\right)}^{\phi}\right)\left(\phi\left(\boldsymbol{x}_{i}^{p}\right)-\boldsymbol{\mu}_{\left(\boldsymbol{x}_{i}^{p}, i\right)}^{\phi}\right)^{\mathrm{T}} .
\end{gathered}
$$

Thirdly, the aim of KJDA is to look for the transformation $v$ to maximize the scatter of samples from a different class and minimize the scatter of samples from the same class, regardless of whether the training sample is sufficient. Thus, the goal of KJDA can be written as

$$
\boldsymbol{v}=\underset{\boldsymbol{v}}{\arg \max } \frac{\boldsymbol{v}^{\mathrm{T}}\left(\boldsymbol{S}_{G B}^{\phi}+\delta \boldsymbol{S}_{L B}^{\phi}\right) \boldsymbol{v}}{\boldsymbol{v}^{\mathrm{T}}\left(\boldsymbol{S}_{G W}^{\phi}+\delta \boldsymbol{S}_{L W}^{\phi}\right) \boldsymbol{v}}, \quad \delta>0
$$

where $\delta$ is a non-negative coefficient endowing different weights to global and local terms for enhancing recognition performance. When $\delta$ approaches 0 , KJDA is estimated by $\arg \max \boldsymbol{v}^{\mathrm{T}} \boldsymbol{S}_{G B}^{\phi} \boldsymbol{v} / \boldsymbol{v}^{\mathrm{T}} \boldsymbol{S}_{G W}^{\phi} \boldsymbol{v}$, which is just equivalent to KLDA; when $\delta$ approaches $+\infty$, KJDA is approximated by $\arg \max \boldsymbol{v}^{\mathrm{T}} \boldsymbol{S}_{L B}^{\phi} \boldsymbol{v} / \boldsymbol{v}^{\mathrm{T}} \boldsymbol{S}_{L W}^{\phi} \boldsymbol{v}$, which is similar to KLSMDA. A proper value of $\delta$ helps to establish a suitable mean model criterion for HRRP target recognition, thereby improving the accuracy of the target recognition while reducing dimensionality. Then by kernel tricks and the theory of reproducing kernels [7], the objective function in (14) can be described as

$$
\boldsymbol{\alpha}=\underset{\boldsymbol{\alpha}}{\arg \max } \frac{\boldsymbol{\alpha}^{\mathrm{T}}\left(\boldsymbol{K}_{G B}+\delta \boldsymbol{K}_{L B}\right) \boldsymbol{\alpha}}{\boldsymbol{\alpha}^{\mathrm{T}}\left(\boldsymbol{K}_{G W}+\delta \boldsymbol{K}_{L W}\right) \boldsymbol{\alpha}}
$$

where $\boldsymbol{K}_{G B}, \boldsymbol{K}_{L B}, \boldsymbol{K}_{G W}$ and $\boldsymbol{K}_{L W}$ can be calculated as

$$
\begin{aligned}
& \boldsymbol{K}_{G B}=\sum_{i=1}^{C}\left(\boldsymbol{m}_{i}-\boldsymbol{m}\right)\left(\boldsymbol{m}_{i}-\boldsymbol{m}\right)^{\mathrm{T}} \\
& \boldsymbol{K}_{L B}=\sum_{i=1}^{C} \sum_{p=1}^{N_{i}} \sum_{j=1, j \neq i}^{C} \gamma_{B\left(\boldsymbol{x}_{i}^{p}, j\right)}^{\phi} . \\
& \left(\boldsymbol{\xi}_{\boldsymbol{x}_{i}^{p}}-\boldsymbol{m}_{\left(\boldsymbol{x}_{i}^{p}, j\right)}\right)\left(\boldsymbol{\xi}_{\boldsymbol{x}_{i}^{p}}-\boldsymbol{m}_{\left(\boldsymbol{x}_{i}^{p}, j\right)}\right)^{\mathrm{T}} \\
& \boldsymbol{K}_{G W}=\sum_{i=1}^{C} \sum_{p=1}^{N_{i}}\left(\boldsymbol{\xi}_{\boldsymbol{x}_{i}^{p}}-\boldsymbol{m}_{i}\right)\left(\boldsymbol{\xi}_{\boldsymbol{x}_{i}^{p}}-\boldsymbol{m}_{i}\right)^{\mathrm{T}} \\
& \boldsymbol{K}_{L W}=\sum_{i=1}^{C} \sum_{p=1}^{N_{i}} \gamma_{W\left(\boldsymbol{x}_{i}^{p}, i\right)}^{\phi}\left(\boldsymbol{\xi}_{\boldsymbol{x}_{i}^{p}}-\boldsymbol{m}_{\left(\boldsymbol{x}_{i}^{p}, i\right)}\right) . \\
& \left(\boldsymbol{\xi}_{\boldsymbol{x}_{i}^{p}}-\boldsymbol{m}_{\left(\boldsymbol{x}_{i}^{p}, i\right)}\right)^{\mathrm{T}} .
\end{aligned}
$$

Meanwhile, $\boldsymbol{m}$ and $\boldsymbol{m}_{i}$, respectively, can be expressed as

$$
\begin{gathered}
\boldsymbol{m}=\frac{1}{N} \sum_{i=1}^{C} \sum_{p=1}^{N_{i}} \boldsymbol{\xi}_{\boldsymbol{x}_{i}^{p}} \\
\boldsymbol{m}_{i}=\frac{1}{N_{i}} \sum_{p=1}^{N_{i}} \boldsymbol{\xi}_{\boldsymbol{x}_{i}^{p}} .
\end{gathered}
$$

KLSMDA gives the definitions of $\gamma_{B\left(\boldsymbol{x}_{i}^{p}, j\right)}^{\phi}, \gamma_{W\left(\boldsymbol{x}_{i}^{p}, i\right)}^{\phi}$, $\boldsymbol{m}_{\left(\boldsymbol{x}_{i}^{p}, i\right)}, \boldsymbol{m}_{\left(\boldsymbol{x}_{i}^{p}, j\right)}$ and $\boldsymbol{\xi}_{\boldsymbol{x}_{i}^{p} \text {. }}$

Finally, (15) can be solved by finding the eigenvectors corresponding to the first $d$ largest eigenvalues of $\boldsymbol{K}_{W}^{-1} \boldsymbol{K}_{B}$. Once $\boldsymbol{\alpha}$ is obtained, for any sample $\boldsymbol{x} \in \mathbf{R}^{D}$ in the original space, a $d$-dimensional column vector $\boldsymbol{y}$ can be defined as

$$
\boldsymbol{y}=\boldsymbol{v}^{\mathrm{T}} \phi(\boldsymbol{x})=\boldsymbol{\alpha}^{\mathrm{T}}\left[k\left(\boldsymbol{x}, \boldsymbol{x}_{1}^{1}\right), \ldots, k\left(\boldsymbol{x}, \boldsymbol{x}_{C}^{N_{C}}\right)\right]^{\mathrm{T}} .
$$

\section{Experiments}

The experiments presented in this paper are based on the measured HRRP data for three types of aircraft [15-18], including Cessna Citation S/II, Yark-42 and An-26, and the parameters of the aircraft are given in Table 1 .

Table 1 Parameters of three aircraft

\begin{tabular}{cccc}
\hline Aircraft & Length & Width & Height \\
\hline An-26 & 23.80 & 29.20 & 9.83 \\
Cessna Citation S/II & 14.40 & 15.90 & 4.57 \\
Yark-42 & 36.38 & 34.88 & 9.83 \\
\hline
\end{tabular}

The projections of aircraft trajectories onto the ground are showed in Fig. 1. All trajectories are segmented, every segment has 26000 original HRRP data. Training set consists of the sixth segment of An-26, the fifth segment of Cessna Citation S/II, and the second segment of Yark42. The testing set consists of the seventh segment of An26, the fifth segment of Yark-42, and the sixth segment of 
Cessna Citation S/II. In addition, because of the amplitudescale and time-shift sensitivities of HRRP, the power spectrum of the original HRRP by $l_{2}$-norm normalization is used to perform recognition [19].

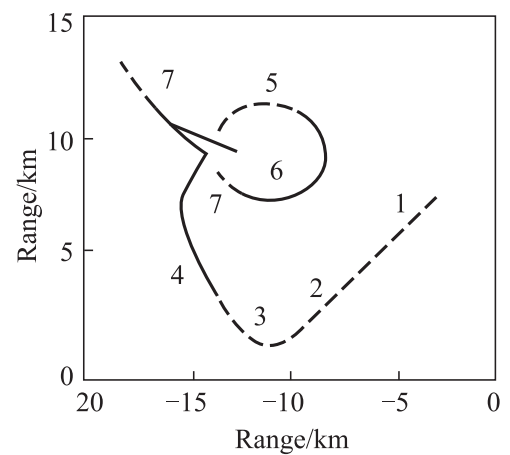

(a) An-26

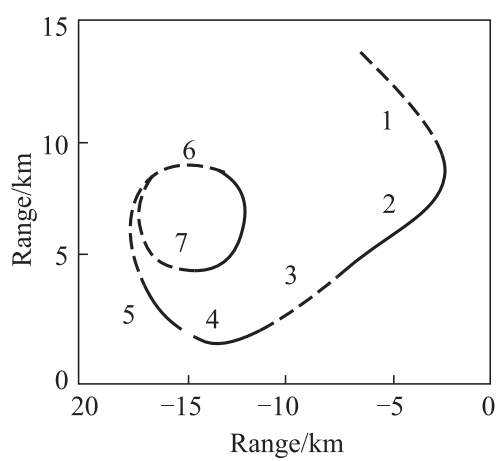

(b) Cessna Citation

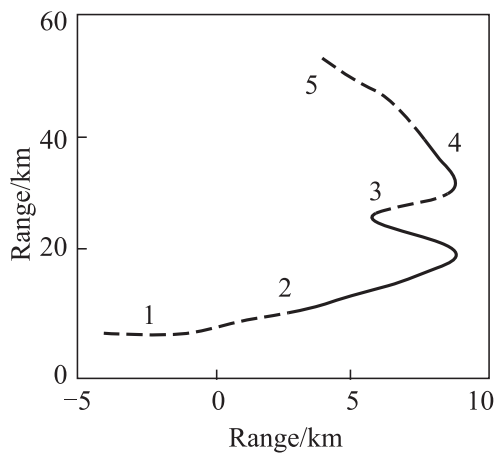

(c) Yark-42

Fig. 1 Projections of aircraft trajectories onto ground

Fig. 2 describes the average recognition performance for the power spectrum using the above six methods: LDA, local sampling mean discriminant analysis (LSMDA) [20], combinatorial discriminant analysis (CDA) [21], KLDA, KLSMDA and KJDA. It is found that the recognition performance of nonlinear methods exceeds that of linear methods in the K-nearest neighbor (KNN) [22] and kernel support vector machine (KSVM) [23]. This is because the nuclear function can further extract useful information from the kernel feature space and well describe the nonlinear structure among targets. However, the reason why recognition accuracy of nonlinear methods by KSVM is lower than that by KNN is that KSVM performs kernel tricks again on the HRRP, thereby causing an overfitting problem.

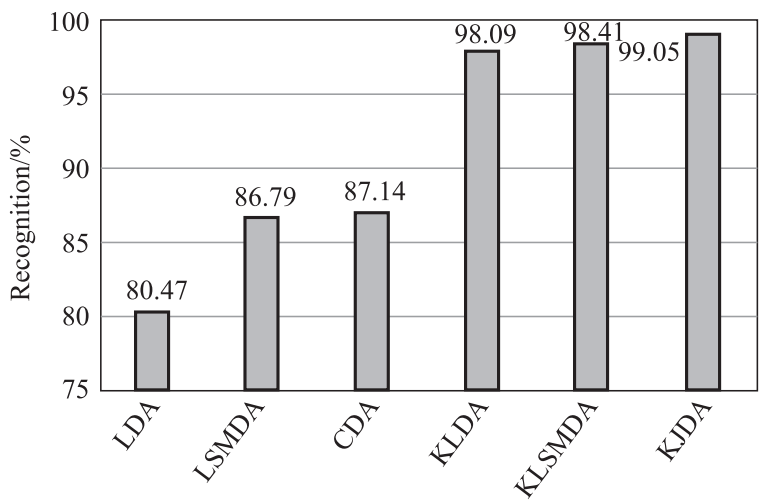

(a) $\mathrm{KNN}$

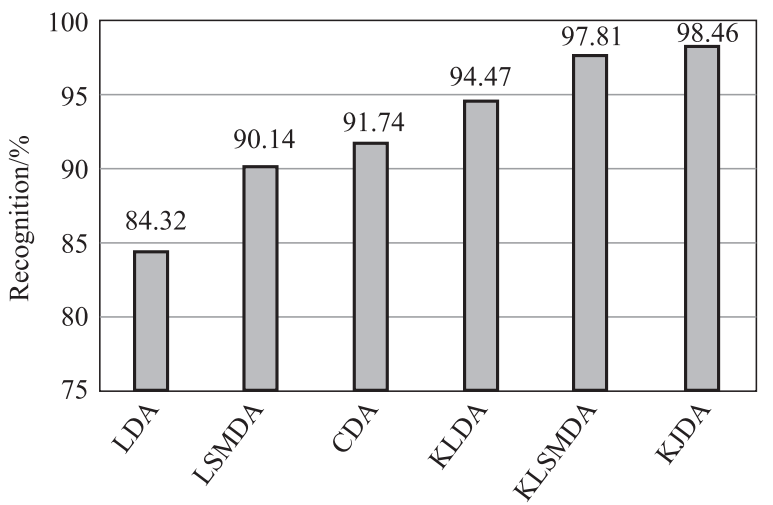

(b) KSVM

Fig. 2 Recognition performance in different methods

Table 2 shows the reduced dimension of the corresponding algorithms. Because the upper bound of the vector dimensionality is $C-1$ in LDA and KLDA algorithms, which leads to partial loss of feature information, the classification accuracy is poor. The LDMA, CDA, KLMDA and KJDA algorithms not only overcome the drawback of low reduced dimension, but also relax requirements of the LDA and KLDA algorithms for data obeying the Gaussian distribution. Compared with the corresponding algorithms, the KJDA algorithm can extract global and local information. It is the reason that the dimension of projection vectors in KJDA is the highest.

Table 2 Reduced dimension of different algorithms

\begin{tabular}{ccccc}
\hline Algorithm & LSMDA & CDA & KLSMDA & KJDA \\
\hline Reduced dimension & 23 & 47 & 29 & 60
\end{tabular}

Fig. 3 shows the HRRP target recognition accuracy with different feature dimensions. From the analysis, the recog- 
nition accuracy of the LSMDA and CDA methods improves with increasing dimensions. However, after a certain dimension, the recognition performance remains almost unchanged. The KLSMDA and KJDA algorithms can simultaneously extract the linear and nonlinear features of the training samples. However, as the feature dimension increases to a certain dimension, the recognition performance is reduced. This is because nonlinear feature extraction methods deeply extract information from the training sample, resulting in overfitting of the training sample in the high-dimensional space, so that the test sample may not have better recognition performance with increasing dimensions.

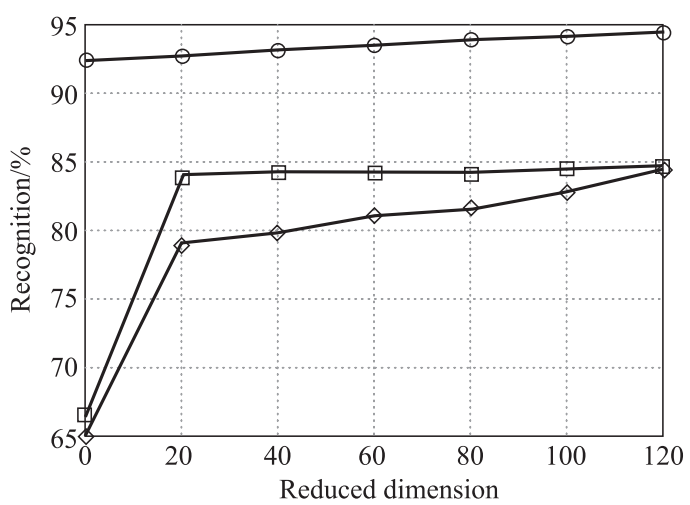

(a) LSMDA

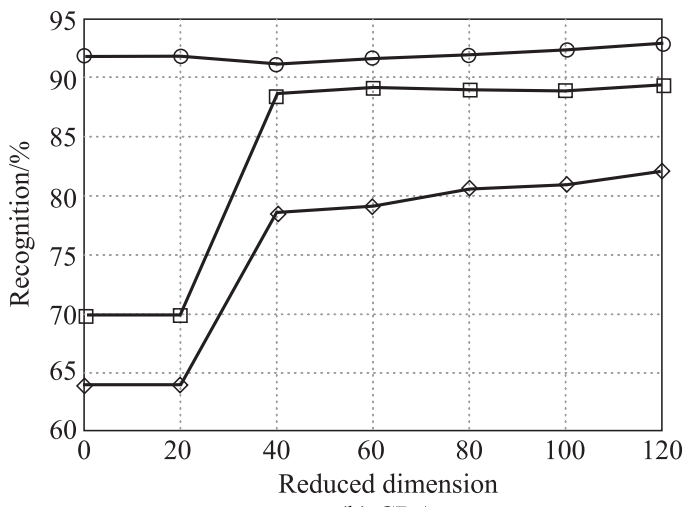

(b) $\mathrm{CDA}$

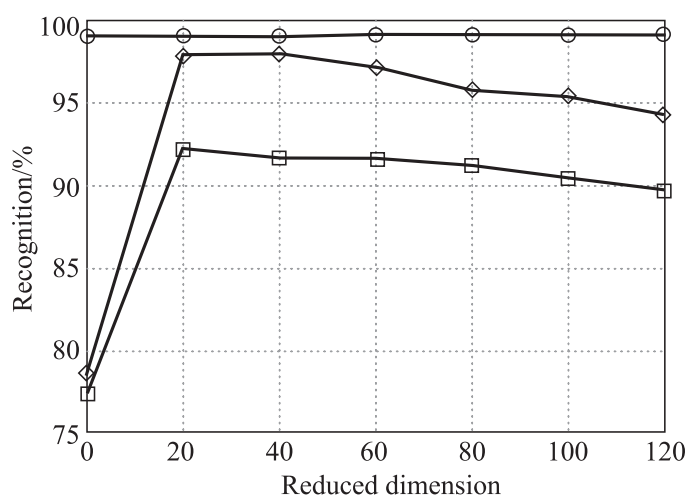

(c) KLSMDA

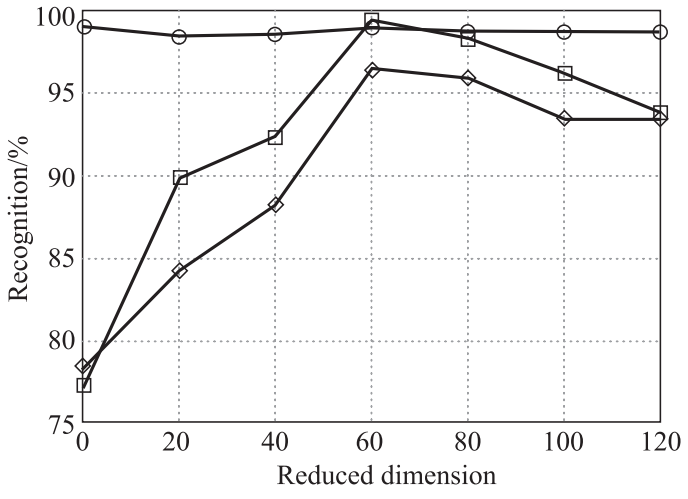

(d) KJDA

๑ :An-26; $\neg:$ Cessna Citation S/II; $\neg:$ Yark-42.

Fig. 3 Recognition accuracy with different dimensions

In order to verify that KJDA can solve sample problems, we select the top $20 \%, 40 \%, 60 \%$ and $80 \%$ of the training set as training data, and then use all of the testing set as testing data to evaluate performances. Experimental results are shown in Table 3. For adequate training samples, the optimal $\delta$ of KJDA approaches $+\infty$, which shows that local terms play a more important role than global ones. For insufficient training samples, KJDA still achieves the best recognition performance. As we all know, insufficient training samples lead to the small sample problem. In this paper, the optimal $\delta$ of KJDA approaching 0 can construct robust scattering matrices, which offers us a prospective method to cope with the small problem in HRRP target recognition.

Table 3 Recognition result on HRRP dataset

$\%$

\begin{tabular}{ccccc}
\hline \multirow{2}{*}{ Algorithm } & \multicolumn{4}{c}{ Recognition via training percentage } \\
\cline { 2 - 5 } & $20 \%$ & $40 \%$ & $60 \%$ & $80 \%$ \\
& $\delta=0.01$ & $\delta=1$ & $\delta=100$ & $\delta=1000$ \\
\hline LDA & 70.38 & 75.73 & 78.83 & 80.06 \\
LSMDA & 68.99 & 75.47 & 83.50 & 85.12 \\
CDA & 70.98 & 77.90 & 86.52 & 86.60 \\
KLDA & 81.06 & 88.65 & 94.88 & 97.76 \\
KLSMDA & 83.42 & 88.70 & 95.04 & 97.83 \\
KJDA & 83.76 & 90.38 & 96.21 & 98.15 \\
\hline
\end{tabular}

\section{Conclusions}

The KJDA algorithm is proposed to overcome the intrinsic drawbacks of LDA, KLDA, LSMDA and KLSMDA. By trading off the contributions between global and local terms, KJDA is exploited to search an appropriate midpoint between global and local criteria to construct robust scattering matrices, and hence is expected to extract potential information in the high-dimensional space. Compared with other methods in HRRP target recognition, it shows that KJDA is an effective nonlinear feature extraction method. 
Although KJDA performs well in the above experiments, there is still an open problem to be addressed next. There are three free parameters $\sigma_{1}, \sigma_{2}$ and $\delta$ in our work, and it is timing-consuming to properly select them, especially the choice of $\delta$. Besides, detecting an exact nonnegative coefficient $\delta$ to endow different weights to global and local terms can provide a good discriminability in different targets. Consequently, how to design a simple and efficient feature extraction method to be applied in the actual situation is still a challenge.

\section{References}

[1] GAO F, MEI J Y, SUN J P, et al. Target detection and recognition in SAR imagery based on KFDA. Journal of Systems Engineering and Electronics. 2015, 26(4): 720-731.

[2] BAI X, ZHOU F, BAO Z, et al. High-resolution range profiles. IEEE Trans. on Geoscience and Remote Sensing, 2014, 52(5): $2369-2381$.

[3] LIU H W, FENG B, CHEN B, et al. Radar high-resolution range profiles target recognition based on stable dictionary learning. IET Radar, Sonar and Navigation, 2016, 10(1): 228 237.

[4] GUO Y, XIAO H T, KAN Y Z, et al. Learning using privileged information for HRRP-based radar target recognition. IET Signal Processing, 2018, 12(2): $188-197$.

[5] NATALIA A, JOSEPH A. Thresholding method for dimensionality reduction in recognition systems. IEEE Trans. on Information Theory, 2001, 47(7): 2903-2920.

[6] GARZON-GUERRERO J A, RUIZ D P, CARRION M C. Classification of geometrical targets using natural resonances and principal components analysis. IEEE Trans. on Antennas and Propagation, 2013, 61(9): $4881-4884$.

[7] KARI T, GAO W. Power transformer fault diagnosis using FCM and improved PCA. The Journal of Engineering, 2017, 14: $2605-2608$.

[8] FU J S, LIAO K, YANG W L. Radar HRRP target recognition using multi-KFD-based LDA algorithm. Progress in Electromagnetics Research C, 2012, 30: 15-26.

[9] BELHUMEUR P N, HESPANHA J P, KRIEGMAN D J. Eigenfaces vs. Fisherfaces: recognition using class specific linear projection. IEEE Trans. on Pattern Analysis and Machine Intelligence, 1997, 19(7): 711-720.

[10] MIKA S, RATSCH G, WESTON J, et al. Fisher discriminant analysis with kernels. Proc. of the IEEE Neural Networks for Signal Processing IX, 1999: 41-48.

[11] HU W, HU H. Heterogeneous face recognition based on modality-independent kernel Fisher discriminant analysis joint sparse auto-encoder. Electronics Letters, 2016, 52(21): 1753 1755.

[12] FENG G Y, XIAO H T, FU Q. Local sampling mean discriminant analysis with kernels. Electronics Letters, 2012, 48(1): $22-23$.

[13] JUWEI L, PLATANIOTIS K N, VENETSANOPOULOS A N. Face recognition using kernel direct discriminant analysis algorithms. IEEE Trans. on Neural Networks, 2003, 14(1): 117 126.

[14] YOU D, HAMSICI O C, MARTINEZ A M. Kernel optimization in discriminant analysis. IEEE Trans. on Pattern Analysis \& Machine Intelligence, 2010, 33(3): 631-638.

[15] PAN M, DU L, WANG P H, et al. Noise-robust modification method for Gaussian-based models with application to radar HRRP recognition. IEEE Geoscience and Remote Sensing Letters, 2013, 10(3): $662-668$.
[16] BAI X, ZHOU F, BAO Z, et al. High-resolution radar imaging of space targets based on HRRP series. IEEE Trans. on Geoscience and Remote Sensing, 2014, 52(5): 2369-2381.

[17] GUO Y, XIAO H, FU Q. Least square support vector data description for HRRP-based radar target recognition. Applied Intelligence, 2017, 46(2): 365-372.

[18] PAN M, JIANG J, KONG Q, et al. Radar HRRP target recognition based on t-SNE segmentation and discriminant deep belief network. IEEE Geoscience and Remote Sensing Letters, 2017, 14(9): $1609-1613$.

[19] ZHANG H, DING D, FAN Z, et al. Adaptive neighborhoodpreserving discriminant projection method for HRRP-based radar target recognition. IEEE Antennas and Wireless Propagation Letters, 2015, 14: 650-653.

[20] CHAI J, LIU H, BAO Z. Generalized re-weighting local sampling mean discriminant analysis. Pattern Recognition, 2010, 43(10): $3422-3432$.

[21] CHAI J, LIU H, BAO Z. Combinatorial discriminant analysis: supervised feature extraction that integrates global and local criteria. Electronics Letters, 2009, 45(18): 934-935.

[22] ALTMAN N S. An introduction to kernel and nearest-neighbor nonparametric regression. American Statistician, 1992, 46(3): $175-185$.

[23] GAO L P, ZHANG X L, GAO G P, et al. Fusion image based radar signal feature extraction and modulation recognition. IEEE Access, 2019, 7: 13135-13148.

\section{Biographies}

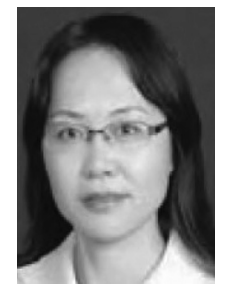

LIU Wenbo was born in 1968. She received her Ph.D. degree from Nanjing University of Aeronautics and Astronautics in 2002. She is a professor in Nanjing University of Aeronautics and Astronautics. Her research interests are signal processing and pattern recognize.

E-mail: wenboliu@nuaa.edu.cn

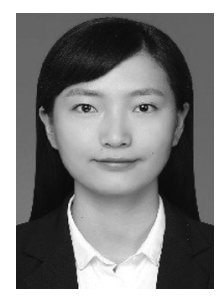

YUAN Jiawen was born in 1994. She is a Ph.D. candidate in Nanjing University of Aeronautics and Astronautics. Her research interests include radar signal processing and target recognition.

E-mail: yuanjiawen@nuaa.edu.cn

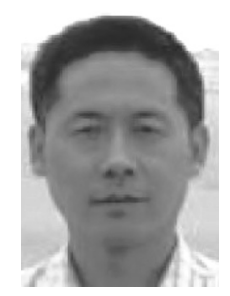

ZHANG Gong was born in 1964. He received his Ph.D. degree from Nanjing University of Aeronautics and Astronautics in 2002. He is a professor in Nanjing University of Aeronautics and Astronautics. His research interests are SAR image processing, target detection and target recognition.

E-mail: gzhang@nuaa.edu.cn

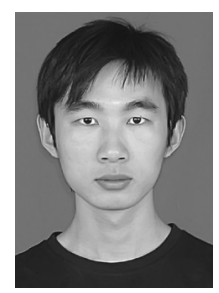

SHEN Qian was born in 1987. He is a Ph.D. candidate in Nanjing University of Aeronautics and Astronautics. His research interests are image encryption, compressive sensing and chaos theory. E-mail: qianshen@nuaa.edu.cn 